PROCEEDINGS OF THE

AMERICAN MATHEMATICAL SOCIETY

Volume 140, Number 12, December 2012, Pages 4359-4368

S 0002-9939(2012)11292-4

Article electronically published on May 1, 2012

\title{
COVERING AN UNCOUNTABLE SQUARE BY COUNTABLY MANY CONTINUOUS FUNCTIONS
}

\author{
WIESŁAW KUBIŚ AND BENJAMIN VEJNAR
}

(Communicated by Julia Knight)

\begin{abstract}
We prove that there exists a countable family of continuous real functions whose graphs, together with their inverses, cover an uncountable square, i.e. a set of the form $X \times X$, where $X \subseteq \mathbb{R}$ is uncountable. This extends Sierpiński's theorem from 1919, saying that $S \times S$ can be covered by countably many graphs of functions and inverses of functions if and only if $|S| \leqslant \aleph_{1}$. Using forcing and absoluteness arguments, we also prove the existence of countably many 1-Lipschitz functions on the Cantor set endowed with the standard non-archimedean metric that cover an uncountable square.
\end{abstract}

\section{INTRODUCTION}

A classical result of Sierpiński from 1919 (see [11, 12] or [13, Chapter I]) says that, given a set $S$ of cardinality $\aleph_{1}$, there exists a countable family of functions $f_{n}: S \rightarrow S$ such that

$$
S \times S=\bigcup_{n \in \omega}\left(f_{n} \cup f_{n}^{-1}\right),
$$

where $f_{n}^{-1}$ is the inverse of $f_{n}$, i.e. $f_{n}^{-1}=\left\{\left\langle f_{n}(x), x\right\rangle: x \in S\right\}$. A typical proof proceeds as follows. Assume $S=\omega_{1}$, and for each positive $\beta<\omega_{1}$ choose a surjection $g_{\beta}: \omega \rightarrow \beta$. Define $f_{n}: \omega_{1} \rightarrow \omega_{1}$ by the equation $f_{n}(\beta)=g_{\beta}(n)$. For every $\langle\alpha, \beta\rangle \in S \times S$ with $\alpha<\beta$ there exists $n$ such that $g_{\beta}(n)=\alpha$; thus $\langle\alpha, \beta\rangle \in f_{n}$ and $\langle\beta, \alpha\rangle \in f_{n}^{-1}$. Finally, it suffices to add the identity function to the family $\left\{f_{n}\right\}_{n \in \omega}$ in order to get (1.1). It is worth saying that the sets $f_{n}^{-1}(\alpha)$ form an Ulam matrix on $\omega_{1}$. See e.g. [4, Chapter 10] or [8, Chapter II, §6] for applications of Ulam matrices.

An easy argument (also noted by Sierpiński) shows that the above statement fails for a set $S$ of cardinality $\aleph_{2}$. In particular, the continuum hypothesis is equivalent to the statement "there exists a countable family of functions which, together with their inverses, cover the plane."

Received by the editors January 11, 2010 and, in revised form, June 6, 2011.

2010 Mathematics Subject Classification. Primary 03E05, 03E15; Secondary 54H05.

Key words and phrases. Uncountable square, covering by continuous functions, set of cardinality $\aleph_{1}$.

The research of the first author was supported in part by Grant IAA 100190901 and by the Institutional Research Plan of the Academy of Sciences of Czech Republic, No. AVOZ 101905 03.

The research of the second author was supported by Grant SVV-2011-263316 of the Czech Republic Ministry of Education, Youth and Sports.

(C)2012 American Mathematical Society Reverts to public domain 28 years from publication 
Let us say that a set $M$ is covered by a family of functions $\mathcal{F}$ if for every $\langle x, y\rangle \in M$ there is $f \in \mathcal{F}$ such that either $y=f(x)$ or $x=f(y)$.

Question 1. Does there exist a sequence $\left\{f_{n}: \mathbb{R} \rightarrow \mathbb{R}\right\}_{n \in \omega}$ of continuous functions that covers an uncountable square?

One can hope for a positive answer only when the side $S$ of the square has some smallness properties, besides having cardinality $\aleph_{1}$. In fact, by a result of Zakrzewski [14, Theorem 2.1], if $S \times S$ is covered by countably many functions (and their inverses) whose graphs are Borel sets, then $S$ is universally small; i.e., $S$ belongs to every Borel $\sigma$-ideal $I \subseteq \mathcal{P}(\mathbb{R})$ such that Borel $(\mathbb{R}) / I$ satisfies the countable chain condition.

Actually, consistent affirmative answers to Question 1 already exist in the literature. Namely, Abraham and Geschke 1 showed that for every set $X \subseteq \mathbb{R}$ of cardinality $\aleph_{1}$ there is a ccc forcing notion adding countably many continuous functions that cover $X \times X$. Consequently, under Martin's Axiom every $\aleph_{1}$-square in the plane is covered by a countable family of continuous functions. The Open Coloring Axiom of Abraham, Rubin and Shelah 2 implies that for every set $X \subseteq 2^{\omega}$ of size $\aleph_{1}$ there is a countable family of 1-Lipschitz functions that covers $X \times X$ (see [3] for more details).

Yet another motivation for addressing Question 1 comes from the work of Shelah [10], continued in [7, where planar Borel sets without perfect squares were studied (a perfect square is, by definition, a set of the form $C \times C$, where $C$ is perfect, i.e. nonempty, compact, without isolated points). It is not hard to prove (see e.g. 6, Thm. 2.2]) that a $G_{\delta}$ subset of the plane containing countable squares of arbitrarily large countable Cantor-Bendixson ranks also contains a perfect square. On the other hand, using Keisler's completeness, it has been proved in [10] that there exists in ZFC a planar $F_{\sigma}$ set $C$ such that $S \times S \subseteq C$ for some uncountable set $S$, while $P_{0} \times P_{1} \nsubseteq \subseteq C$ whenever $P_{0}, P_{1}$ are perfect sets. A significant part of 7 is devoted to a ZFC construction of certain $F_{\sigma}$ sets in the plane that do not contain perfect squares, while consistently they contain squares of a prescribed cardinality below $\aleph_{\omega_{1}}$. These sets moreover have a certain universality property among sets of the same type (see [7] for details). Because of the results of [10] and [7, it is natural to ask for the existence of a more special planar $F_{\sigma}$ set that covers an uncountable square, namely, a set consisting of countably many continuous real functions and their inverses. There are natural restrictions here. Namely, such a set cannot contain rectangles of the form $S_{0} \times S_{1}$, where $\left|S_{i}\right|=\aleph_{1}$ and $\left|S_{1-i}\right| \geqslant \aleph_{2}$. Easy absoluteness arguments show that it cannot contain perfect rectangles; therefore the best property we can expect is covering a square of cardinality $\aleph_{1}$.

In the present paper we prove the following results:

(I) There exists a family of continuous functions $\mathcal{F}=\left\{f_{n}: 2^{\omega} \rightarrow 2^{\omega}\right\}_{n \in \omega}$ such that every maximal square covered by $\mathcal{F}$ is uncountable (Theorem 2.1 below).

(II) There exists a countable family of 1-Lipschitz functions on $2^{\omega}$ that covers an uncountable square (Theorem 3.1 below).

In the statements above, $2^{\omega}$ denotes the Cantor set endowed with the standard non-archimedean metric. The proof of Theorem 2.1 is very short and elementary; however the functions $f_{n}$ are not Lipschitz with respect to any natural metric on $2^{\omega}$. In order to prove Theorem 3.1, we first describe a natural ccc forcing notion that 
introduces the required family of 1-Lipschitz functions together with the required uncountable square. Second, using Keisler's completeness theorem [5], we deduce that such a family exists in ZFC. We do not know any direct construction.

We also observe (Theorem 4.1 below) that it is impossible to cover the square of any uncountable compact Hausdorff space by countably many continuous functions and their inverses.

Finally, let us mention a very recent work of Kunen [9] which deals with the question of covering an uncountable square by differentiable functions. In particular, 9, Thm. 1.2] says that there exists an uncountable subset of the real line whose square is covered by countably many smooth real functions. This is a ZFC result with a direct (although quite technical) proof. The paper [9] also contains interesting consistency results related to the question of whether every square of size $\aleph_{1}$ can be covered by countably many functions satisfying natural smoothness conditions.

\section{MAIN RESULT}

Theorem 2.1. Let $X$ be a topological space containing at least two points and suppose there exists a continuous onto mapping $\varphi: X \rightarrow X^{\omega}$. Then there exists a countable family of continuous mappings of $X$ into itself such that every maximal square covered by this family is uncountable.

Proof. We first note that there is a continuous mapping $f$ from $X$ onto $X^{\omega}$ with the property that the pre-image of every sequence in $X^{\omega}$ is uncountable. Indeed, consider the projection $\pi_{0}: X^{\omega} \rightarrow X$ which takes the sequence $\left\langle s_{i}: i \in \omega\right\rangle \in X^{\omega}$ to the first entry $s_{0} \in X$. Then form the composition $f=\varphi \circ \pi_{0} \circ \varphi: X \rightarrow X^{\omega}$. Now if $\bar{y} \in X^{\omega}$ and $s_{0} \in X$ are such that $\varphi\left(s_{0}\right)=\bar{y}$, then $f(x)=\bar{y}$ whenever the sequence $\varphi(x)$ begins with $s_{0}$. But there are (at least) continuum many such sequences, as $|X| \geq 2$. Hence the pre-image $f^{-1}(\bar{y})$ is uncountable.

Now define $f_{n}: X \rightarrow X$ for $n \in \omega$ by $f_{n}(x)=\pi_{n}(f(x))$, where $\pi_{n}: X^{\omega} \rightarrow X$ is the $n$-th entry projection. It remains to show that the system $\left\{f_{n}: n \in \omega\right\}$ together with the identity function on $X$ is the desired family. Take any countable set $S=\left\{s_{n}: n \in \omega\right\} \subseteq X$ whose square is covered by our family of functions. We will show that it is not maximal; that is, we extend $S$ to a set whose square is still covered by our family. Simply take an arbitrary point $x$ from the uncountable set $f^{-1}\left(\left\langle s_{0}, s_{1}, \ldots\right\rangle\right)$ that is not in $S$. Then the square of the set $S \cup\{x\}$ is covered by our family of functions since $f_{n}(x)=s_{n}$ for every $n \in \omega$ and $\operatorname{id}_{X}(x)=x$.

Note that the empty set is trivially covered by any family of functions so that by the Kuratowski-Zorn lemma it follows that there exists a maximal set $S \subseteq X$ whose square is covered by our family. Hence we get an uncountable set whose square is covered by a countable family of continuous functions. Thus the axiom of choice and the fact that the Cantor space is homeomorphic to its countable power yield the following corollary.

Corollary 2.2. There exists a family of continuous functions of the Cantor space to itself that covers an uncountable square.

This answers Question 1, since any continuous function of the Cantor space to itself can be extended to a continuous real function. 


\section{FORCING COUNTABLY MANY LiPSCHITZ FUNCTIONS}

It is easy to see that the functions constructed in Theorem 2.1 are not Lipschitz with respect to the natural metric on the Cantor set. We do not know a direct construction of a countable family of 1-Lipschitz functions covering an uncountable square. This section is devoted to showing that such a family can be introduced by a natural forcing notion. Using Keisler's completeness, we later conclude that this family exists in ZFC, namely:

Theorem 3.1. There exists a countable family of 1-Lipschitz functions on the Cantor set that covers an uncountable square.

We first show the consistency of the above statement with the axioms of ZFC. The metric on $2^{\omega}$ which we have in mind is given by the formula $d(x, y)=2^{-k}$, where $k$ is the smallest natural number such that $x \uparrow k \neq y \uparrow k$.

Given a natural number $n$, we shall denote by $2^{n}$ the complete binary tree consisting of all zero-one sequences of length $n$. Trees of the form $2^{n}$ serve as finite approximations of the Cantor set $2^{\omega}$. We consider $2^{n}$ with the lexicographic ordering and with the metric defined above, as in the case of $2^{\omega}$. Denote by $\mathbb{L}_{1}(n)$ the set of all 1-Lipschitz functions of the form $g: 2^{n} \rightarrow 2^{n}$. Note that $g$ is 1-Lipschitz iff the length of the splitting point of $g(a)$ and $g(b)$ (where $a \neq b$ are in $2^{n}$ ) is not shorter than the length of the splitting point of $a$ and $b$.

We are going to define a forcing notion $\mathbb{P}$ which will introduce a countable family of Lipschitz functions covering an uncountable square.

A condition $p \in \mathbb{P}$ is, by definition, of the form $p=\left\langle n^{p}, s^{p}, v^{p}, \mathcal{F}^{p}, \gamma^{p}, \varrho^{p}\right\rangle$, where:

(1) $n^{p} \in \omega, s^{p} \in[\omega]^{<\omega}$ and $v^{p} \in\left[\omega_{1}\right]^{<\omega}$;

(2) $\mathcal{F}^{p}=\left\{f_{i}^{p}\right\}_{i \in s^{p}} \subseteq \mathbb{L}_{1}\left(n^{p}\right)$ and $\varrho^{p}:\left[v^{p}\right]^{2} \rightarrow s^{p}$;

$\left(2^{\prime}\right) \varrho^{p}(\alpha, \beta) \neq \varrho^{p}\left(\alpha^{\prime}, \beta\right)$ whenever $\alpha<\alpha^{\prime}<\beta$;

(3) $\gamma^{p}: v^{p} \rightarrow 2^{n^{p}}$ is one-to-one;

(4) $\gamma^{p}(\alpha)=f_{\varrho^{p}(\alpha, \beta)}^{p}\left(\gamma^{p}(\beta)\right)$ whenever $\alpha<\beta$ and $\alpha, \beta \in v^{p}$.

Note that condition $\left(2^{\prime}\right)$ is actually implied by the conjunction of $(3)$ and $(4)$. The order of $\mathbb{P}$ is defined naturally. Namely, $p \leqslant q(q$ is stronger than $p)$ iff:

(5) $n^{p} \leqslant n^{q}, s^{p} \subseteq s^{q}, v^{p} \subseteq v^{q}$;

(6) $f_{i}^{q}(\eta) \uparrow n^{p}=f_{i}^{p}\left(\eta\left\lceil n^{\bar{p}}\right)\right.$ for each $i \in s^{p}$ and for every $\eta \in 2^{n^{q}}$;

(7) $\gamma^{q}(\alpha) \uparrow n^{p}=\gamma^{p}(\alpha)$ for every $\alpha \in v^{p}$;

(8) $\varrho^{q} \uparrow\left[v^{p}\right]^{2}=\varrho^{p}$.

It is easy to see (the details are given below) that the forcing $\mathbb{P}$ introduces a countable family $\left\{f_{n}\right\}_{n \in \omega}$ of continuous functions on the Cantor set together with a function $\varrho:\left[\omega_{1}\right]^{2} \rightarrow \omega$ and a one-to-one function $\gamma: \omega_{1} \rightarrow 2^{\omega}$ such that $\gamma(\alpha)=$ $f_{\varrho(\alpha, \beta)}(\gamma(\beta))$ for every $\alpha<\beta<\omega_{1}$. We need to prove that $\mathbb{P}$ does not collapse $\aleph_{1}$.

Lemma 3.2. $\mathbb{P}$ satisfies the countable chain condition.

Proof. Fix a family $\mathcal{G} \subseteq \mathbb{P}$ with $|\mathcal{G}|=\aleph_{1}$. Replacing $\mathcal{G}$ by an uncountable subfamily, we may assume that there exist $n \in \omega, s \in[\omega]^{<\omega}$ and $\mathcal{F}=\left\{f_{i}\right\}_{i \in s} \subseteq \mathbb{L}_{1}(n)$ such that $n^{p}=n, s^{p}=s$ and $\mathcal{F}^{p}=\mathcal{F}$ for every $p \in \mathcal{G}$. Further, refining $\mathcal{G}$, we may assume the following:

(9) $\left\{v^{p}: p \in \mathcal{G}\right\}$ forms a $\Delta$-system with root $a \subseteq \omega_{1}$.

(10) For every $p, q \in \mathcal{G}$ the structures $\left\langle v^{p}, \gamma^{p}, \varrho^{p},<\right\rangle$ and $\left\langle v^{q}, \gamma^{q}, \varrho^{q},<\right\rangle$ are isomorphic, where $<$ is the linear order inherited from $\omega_{1}$. In other words, 
there exists an order preserving bijection $\varphi: v^{p} \rightarrow v^{q}$ such that $\gamma^{p}(\alpha)=$ $\gamma^{q}(\varphi(\alpha))$ and $\varrho^{p}(\alpha, \beta)=\varrho^{q}(\varphi(\alpha), \varphi(\beta))$ for every $\alpha, \beta \in v^{p}$.

For the remaining part of the proof we fix $p, q \in \mathcal{G}$ such that $\max (a)<\min \left(v^{p} \backslash a\right)$ and $\max \left(v^{p}\right)<\min \left(v^{q} \backslash a\right)$. Our aim is to construct $r \in \mathbb{P}$ with $p \leqslant r$ and $q \leqslant r$.

Define $n^{r}=n+1$ and $v^{r}=v^{p} \cup v^{q}$. Note that by (10), $\varrho^{p}, \varrho^{q}$ coincide on $a=v^{p} \cap v^{q}$. Extend $\varrho^{p} \cup \varrho^{q}$ to a function $\varrho^{r}:\left[v^{r}\right]^{2} \rightarrow \omega$ in such a way that $\varrho^{r}$ restricted to the set

$$
\sigma=\left[v^{r}\right]^{2} \backslash\left(\left[v^{p}\right]^{2} \cup\left[v^{q}\right]^{2}\right)=\left\{\{\alpha, \beta\}: \alpha \in v^{p} \backslash a, \beta \in v^{q} \backslash a\right\}
$$

is a bijection onto $t \subseteq \omega \backslash s$. Clearly, $\varrho^{r}$ satisfies $\left(2^{\prime}\right)$; i.e. $\varrho^{r}(\alpha, \beta) \neq \varrho^{r}\left(\alpha^{\prime}, \beta\right)$ whenever $\alpha<\alpha^{\prime}<\beta$. Define $s^{r}=s \cup t$. Then $\varrho^{r}:\left[v^{r}\right]^{2} \rightarrow s^{r}$. Further, define

$$
\gamma^{r}(\alpha)= \begin{cases}\gamma^{p}(\alpha)^{\frown} 0 & \text { if } \alpha \in v^{p}, \\ \gamma^{q}(\alpha)^{\frown} 1 & \text { if } \alpha \in v^{q} \backslash a .\end{cases}
$$

Observe that $\gamma^{r}: v^{r} \rightarrow 2^{n^{r}}$ is one-to-one. It remains to define $\mathcal{F}^{r}=\left\{f_{i}^{r}\right\}_{i \in s^{r}}$.

If $i \in t$, then we define $f_{i}^{r}$ to be the constant function with value $\gamma^{r}(\alpha)$, where $\alpha \in v^{p} \backslash a, \beta \in v^{q} \backslash a$ are such that $i=\varrho^{r}(\alpha, \beta)$. Note that $\alpha, \beta$ are uniquely determined, so there is no ambiguity here and $f_{i}^{r}$ satisfies (4). Finally, fix $i \in s$, $\eta \in 2^{n}, \varepsilon \in 2$ and define

$$
f_{i}^{r}\left(\eta^{\urcorner} \varepsilon\right)= \begin{cases}f_{i}^{p}(\eta)^{\wedge} \varepsilon & \left(\exists \alpha, \beta \in v^{p} \backslash a\right) \alpha<\beta \wedge i=\varrho^{p}(\alpha, \beta) \wedge \eta=\gamma^{p}(\beta), \\ f_{i}^{p}(\eta)^{\frown} 0 & \text { otherwise. }\end{cases}
$$

In this manner we have completed the definition of $r=\left\langle n^{r}, s^{r}, v^{r}, \mathcal{F}^{r}, \gamma^{r}, \varrho^{r}\right\rangle$. In order to show that $r \in \mathbb{P}$, we need to verify condition (4) only, since conditions (1)-(3) are rather clear.

Fix $\alpha<\beta$ in $v^{r}$ and let $\ell=\varrho^{r}(\alpha, \beta)$. If $\ell \in t$, then $f_{\ell}^{r}$ is constantly equal to $\gamma^{r}(\alpha)$; therefore (4) holds in this case. So assume $\ell \in s$ and let $\eta=\gamma^{r}(\beta) \uparrow n$. We consider the following two cases.

Case 1. $\alpha \in v^{q} \backslash a$.

Notice that also $\beta \in v^{q} \backslash a$, because $\alpha<\beta$. By (10), there exist $\alpha^{\prime}, \beta^{\prime} \in v^{p}$ such that $\varrho^{p}\left(\alpha^{\prime}, \beta^{\prime}\right)=\varrho^{q}(\alpha, \beta)=\ell$ and $\gamma^{p}\left(\beta^{\prime}\right)=\gamma^{q}(\beta)=\eta$. Thus the first possibility in the definition of $f_{\ell}^{r}$ occurs, and we have

$$
f_{\ell}^{r}\left(\gamma^{r}(\beta)\right)=f_{\ell}^{r}\left(\eta^{\frown} 1\right)=f_{\ell}(\eta)^{\frown} 1=\gamma^{q}(\alpha)^{\frown} 1=\gamma^{r}(\alpha) ;
$$

therefore (4) holds.

Case 2. $\alpha \in v^{p}$.

Now $\gamma^{r}(\alpha)=\gamma^{p}(\alpha)^{\frown} 0$ and either $\beta \in v^{p}$ or else $\alpha \in a$ and $\beta \in v^{q}$ (because $\ell \in s$ implies that either $\{\alpha, \beta\} \subseteq v^{p}$ or $\left.\{\alpha, \beta\} \subseteq v^{q}\right)$. Observe that $f_{\ell}^{r}\left(\gamma^{r}(\beta)\right) \uparrow$ $n=\gamma^{r}(\alpha) \uparrow n$ by the definition of $f_{\ell}^{r}$ and by the fact that $p, q \in \mathbb{P}$. Thus, the only possibility for the failure of (4) is that $f_{\ell}^{r}\left(\gamma^{r}(\beta)\right)=\gamma^{p}(\alpha)^{\wedge} 1$. Suppose this is the case. By the definition of $f_{\ell}^{r}$, we conclude that $\gamma^{r}(\beta)=\eta^{\wedge} 1$ and in particular $\beta \in v^{q} \backslash a$ and $\alpha \in a$. Moreover, the first case in the definition of $f_{\ell}^{r}$ occurs, so there exists $\alpha^{\prime}<\beta^{\prime}$ in $v^{p} \backslash a$ such that $\ell=\varrho^{p}\left(\alpha^{\prime}, \beta^{\prime}\right)$ and $\eta=\gamma^{p}\left(\beta^{\prime}\right)$. Let $\varphi: v^{p} \rightarrow v^{q}$ be the bijection appearing in condition (10). In particular, $\gamma^{p}(\beta)=\eta=\gamma^{q}\left(\varphi\left(\beta^{\prime}\right)\right)$; therefore $\varphi\left(\beta^{\prime}\right)=\beta$, because $\gamma^{q}$ is one-to-one. Further,

$$
\varrho^{r}\left(\varphi\left(\alpha^{\prime}\right), \beta\right)=\varrho^{q}\left(\varphi\left(\alpha^{\prime}\right), \varphi\left(\beta^{\prime}\right)\right)=\varrho^{p}\left(\alpha^{\prime}, \beta^{\prime}\right)=\ell=\varrho^{r}(\alpha, \beta) .
$$


Thus $\varphi\left(\alpha^{\prime}\right)=\alpha$, because $\varrho^{r}$ satisfies $\left(2^{\prime}\right)$. This leads to a contradiction, because $\alpha \in a, \alpha^{\prime} \in v^{p} \backslash a$ and $\varphi\left[v^{p} \backslash a\right]=v^{q} \backslash a$. Thus (4) holds.

We have proved that $r \in \mathbb{P}$. Clearly $p \leqslant r$ and $q \leqslant r$.

Lemma 3.3. Let $k \in \omega$ and $\xi \in \omega_{1}$. The sets

$$
\mathcal{D}(k)=\left\{p \in \mathbb{P}: n^{p} \geqslant k \text { and } k \in s^{p}\right\}, \quad \mathcal{E}(\xi)=\left\{p \in \mathbb{P}: \xi \in v^{p}\right\}
$$

are dense in $\mathbb{P}$.

Proof. Fix $p \in \mathbb{P}$. Define $n^{q}=n^{p}+1, s^{q}=s^{p} \cup\{k\}, v^{q}=v^{p}, \varrho^{q}=\varrho^{p}, \gamma^{q}(\eta)=$ $\gamma^{p}(\eta)^{\frown} 0$ and $f_{i}^{q}\left(\eta^{\frown} \varepsilon\right)=f_{i}^{p}(\eta)^{\wedge} \varepsilon$ for $i \in s^{p}, \eta \in 2^{n^{p}}, \varepsilon \in 2$. Finally, if $k \notin s^{p}$, let $f_{k}^{q}$ be any function from $\mathbb{L}_{1}\left(n^{p}+1\right)$. In this way we have extended $p$ to a condition $q=\left\langle n^{q}, s^{q}, v^{q}, \mathcal{F}^{q}, \gamma^{q}, \varrho^{q}\right\rangle \in \mathbb{P}$ so that $n^{q}>n^{p}, k \in s^{q}$. Repeating this procedure finitely many times we obtain $r \geqslant p$ such that $n^{r} \geqslant k$ and $k \in s^{r}$. This shows that $\mathcal{D}(k)$ is dense in $\mathbb{P}$.

In order to show the density of $\mathcal{E}(\xi)$, again fix $p \in \mathbb{P}$ and assume $\xi \notin v^{p}$. Define $n^{q}=n^{p}+1$ and $v^{q}=v^{p} \cup\{\xi\}$. Let $\sigma=\left\{\{\xi, \alpha\}: \alpha \in v^{p}\right\}$. Extend $\varrho^{p}$ to a function $\varrho^{q}:\left[v^{q}\right]^{2} \rightarrow \omega$ so that $\varrho^{q}\left\lceil\sigma\right.$ is one-to-one onto $t \subseteq \omega \backslash s^{p}$. Let $s^{q}=s^{p} \cup t$. Further, define $\gamma^{q}(\alpha)=\gamma^{p}(\alpha)^{\wedge} 0$ for $\alpha \in v^{p}$ and let $\gamma^{q}(\xi)$ be the constant one function in $2^{n^{q}}$. It remains to define $\mathcal{F}^{q}$.

Given $i \in s^{p}$, define $f_{i}^{q}\left(\eta^{\curlyvee} \varepsilon\right)=f_{i}^{p}(\eta)^{\frown} \varepsilon$ for every $\eta \in 2^{n^{p}}, \varepsilon \in 2$. Fix $i \in t$ and let $\alpha \in v^{p}$ be such that $i=\varrho^{q}(\alpha, \xi)$. If $\xi<\alpha$, define $f_{i}^{q}$ to be the constant function with value $\gamma^{q}(\xi)$. If $\alpha<\xi$, define $f_{i}^{q}$ to be the constant function with value $\gamma^{q}(\alpha)$. Observe that conditions (1) - (4) are satisfied; therefore $q=\left\langle n^{q}, s^{q}, v^{q}, \mathcal{F}^{q}, \gamma^{q}, \varrho^{q}\right\rangle \in$ $\mathbb{P}$. It is clear that $p \leqslant q$ and $q \in \mathcal{E}(\xi)$.

Lemma 3.4. The poset $\mathbb{P}$ forces a family $\mathcal{F}=\left\{f_{n}: n \in \omega\right\}$ of 1-Lipschitz functions on the Cantor set $2^{\omega}$ and an uncountable set $X \subseteq 2^{\omega}$ whose square is covered by $\mathcal{F}$.

Proof. Let $G$ be a $\mathbb{P}$-generic filter over a fixed ground model $\mathbb{V}$. Define functions $f_{k}: 2^{\omega} \rightarrow 2^{\omega}(k \in \omega), \gamma: \omega_{1} \rightarrow 2^{\omega}$ and $\varrho:\left[\omega_{1}\right]^{2} \rightarrow \omega$ by the following equations:

$$
\begin{aligned}
f_{k}(x)\left\lceil n^{p}\right. & =f_{k}^{p}\left(x\left\lceil n^{p}\right),\right. \\
\gamma(\alpha)\left\lceil n^{p}\right. & =\gamma^{p}(\alpha), \\
\varrho(\alpha, \beta) & =\varrho^{p}(\alpha, \beta),
\end{aligned}
$$

where $x \in 2^{\omega}$ and $p$ is any element of $G$ such that $\alpha, \beta \in v^{p}$ and $k \in s^{p}$. The fact that $G$ is a filter, together with the density of sets $\mathcal{D}(k)$ and $\mathcal{E}(\xi)$ (Lemma 3.3), implies that the above definitions are correct. Let $X=\left\{\gamma(\xi): \xi<\omega_{1}\right\}$. By the definition of $\mathbb{P}$, the set $X \subseteq 2^{\omega}$ is uncountable, the functions $f_{k}$ are 1-Lipschitz and for every $\alpha<\beta<\omega_{1}$ we have that $\gamma(\alpha)=f_{\varrho(\alpha, \beta)}(\gamma(\beta))$. It follows that $X^{2} \subseteq\left\{\operatorname{id}_{2^{\omega}}\right\} \cup \bigcup_{n \in \omega}\left(f_{n} \cup f_{n}^{-1}\right)$.

Proof of Theorem 3.1. The forcing described above shows that the existence of a countable family of 1-Lipschitz functions on the Cantor set covering an uncountable square is consistent with ZFC. We are going to argue, using Keisler's completeness theorem [5], that such a family actually exists in ZFC.

To be more precise, we shall use the completeness theorem for the logic $L^{\omega}(Q)$; see Corollary 3.10 in [5. Here, $L^{\omega}$ stands for the $\omega$-logic, which is an extension of the classical predicate logic with identity by adding a unary predicate symbol $N$ and constant symbols $0,1,2, \ldots$, which are supposed to denote natural numbers. The meaning of $N(x)$ is " $x$ is a natural number". The letter $Q$ stands for a new quantifier 
that means "there exist uncountably many". For the completeness theorem, the language is assumed to be countable; i.e. only countably many functions, relations and constant symbols are allowed. A standard model for $L^{\omega}(Q)$ is a model $M$ of $L^{\omega}(Q)$ in which constant symbols $0,1,2, \ldots$ are interpreted as the "real" natural numbers (in particular $\omega \subseteq M$ ). Further, $M \models N(x)$ if and only if $x \in \omega$, and $M$ satisfies $(Q x) \varphi$ if and only if the set

$$
\{t \in M: M \models \varphi[t]\}
$$

is uncountable, where $\varphi[t]$ is obtained from $\varphi$ by replacing each free occurrence of $x$ by $t$. The logic $L^{\omega}(Q)$ has some natural axioms; we refer the reader to [5] for details. For instance, the following is an axiom:

$$
\neg(Q x) N(x),
$$

which simply says that the set of natural numbers is countable. Finally, $L^{\omega}(Q)$ has three rules of inference: modus ponens, generalization and the $\omega$-rule that allows us to conclude $(\forall x)(N(x) \Longrightarrow \varphi(x))$ from

$$
\varphi(0), \varphi(1), \varphi(2), \ldots
$$

The $\omega$-rule imposes that proofs can have infinite (countable) length. A set of sentences $S$ in $L^{\omega}(Q)$ is consistent if there is no proof of " $0=1$ " from $S$.

Keisler's completeness theorem [5, Cor. 3.10] for $L^{\omega}(Q)$ says that a set of sentences of $L^{\omega}(Q)$ is consistent if and only if it has a standard model.

It should be clear that the property of being consistent in $L^{\omega}(Q)$ is absolute between transitive models of ZFC (in fact, we need downward absoluteness only). Indeed, for a formula $\varphi$, the property of "being provable from a set of sentences $S$ " in $L^{\omega}(Q)$ can be described by defining a suitable ordinal $\operatorname{rank} \mathrm{rk}_{S}$, where $\operatorname{rk}_{S}(\varphi)=0$ if $\varphi \in S$ and $\operatorname{rk}_{S}(\varphi) \leqslant \alpha$ if $\varphi$ is derived from formulae of $\operatorname{rk}_{S}<\alpha$ by using one of the inference rules. Then $\operatorname{rk}_{S}(\varphi)$ is either a countable ordinal or is not defined (precisely when $\varphi$ is not provable from $S$ ). In standard finitary logic, the rank would always be a natural number and infinite values are caused by the $\omega$-rule. It is straightforward that $\operatorname{rk}_{S}$ is absolute. That is, if $\operatorname{rk}_{S}(\varphi)=\alpha$ holds in a fixed transitive model of ZFC containing $S$, then the same holds in any of its transitive ZFC-extensions.

We shall use finitely many predicates and the quantifier $Q$ to describe a countable family of 1-Lipschitz functions on the Cantor set that covers an uncountable square. More precisely, let $C$ and $L$ be unary predicates which will denote elements of the Cantor set and 1-Lipschitz self-maps of the Cantor set respectively. For elements of the Cantor set we need to compute their coordinates, and for maps of the Cantor set we need to compute their values. Therefore we need another two function symbols, $P$ and $V$, where $P(x, k)$ will mean "the $k$-th coordinate of $x$ " and $V(f, x)$ will mean " $f(x)$ ". Finally, we need a binary function symbol $D$ such that, assuming $x, y \in 2^{\omega}$, $D(x, y)$ will denote the minimal $k$ with $x(k) \neq y(k)$. It will be convenient to add the relation symbol $\leqslant$ describing the usual linear ordering of natural numbers.

We now describe the set of sentences $\theta$ saying that $L$ is a countable family of 1-Lipschitz functions on the Cantor set and $C$ is an uncountable set whose square is covered by $L$. The quantifier $Q$ will be needed only for expressing " $L$ is countable" and " $C$ is uncountable".

First of all, let $\theta_{0}$ be a finite set of sentences saying that the sets described by $C$ and $L$ are disjoint and do not consist of natural numbers. Let $\theta_{1}$ be a finite set 
of sentences describing that $P(x, k)$ is $x(k)$ for $x \in 2^{\omega}$ and $k \in \omega$. Namely, the following sentences should be in $\theta_{1}$ :

$$
\begin{gathered}
(\forall x)(\forall k) C(x) \wedge N(k) \Longrightarrow P(x, k)=0 \vee P(x, k)=1, \\
(\forall x, y)(C(x) \wedge C(y) \wedge x \neq y \Longrightarrow(\exists k) N(k) \wedge P(x, k) \neq P(y, k)) .
\end{gathered}
$$

Further, let $\theta_{2}$ be a finite set of sentences describing the meaning of $V(f, x)$ and the fact that $f$ is a 1-Lipschitz map of the Cantor set. The following sentence says that $f$ is 1-Lipschitz:

$$
(\forall x, y, f)(C(x) \wedge C(y) \wedge L(f) \wedge x \neq y)) \Longrightarrow D(V(f, x), V(f, y)) \geqslant D(x, y) .
$$

Let $\theta_{3}$ be a countable set of sentences describing the order $\leqslant$. Namely, $\theta_{3}$ should consist of sentences of the form " $i \leqslant j$ ", where $i, j \in \omega$ are such that $i \leqslant j$ in $\omega$.

Using the predicate $C$ we cannot describe the full Cantor set; however we can add a countable infinite set of sentences $\theta_{4}$ which says that $C$ is dense in the Cantor set. This will ensure that the 1-Lipschitz functions are indeed defined on the full Cantor set, not only on its proper closed subset. So, $\theta_{4}$ should consist of sentences of the form

$$
(\exists x) C(x) \wedge P(x, 0)=s(0) \wedge P(x, 1)=s(1) \wedge \cdots \wedge P(x, n-1)=s(n-1),
$$

where $s \in 2^{n}$ and $n \in \omega$.

Finally, let $\theta_{5}$ consist of the following three sentences:

$$
\begin{aligned}
(\forall x, y) C(x) \wedge C(y) \Longrightarrow & (\exists f) L(f) \wedge(V(f, x)=y \vee V(f, y)=x), \\
& (Q x) C(x), \\
& \neg(Q f) L(f) .
\end{aligned}
$$

These sentences say that the square of $C$ is covered by functions from the set $L$ and, what is most important, $C$ is uncountable while $L$ is countable.

Let $\theta=\theta_{0} \cup \cdots \cup \theta_{5}$. A standard model $M$ of $\theta$ formally consists of $\omega$ and two other disjoint sets $C^{M}$ and $L^{M}$. However, it is obviously isomorphic to a model of the form $\omega \cup C \cup L$, where $C$ is an uncountable dense subset of $2^{\omega}$ and $L$ is a countable family of 1-Lipschitz functions of the Cantor set into itself. Finally, $C \times C$ is covered by $L$.

The forcing arguments described above show that $\theta$ is consistent in some extension of the universe of set theory. Since this property is absolute, $\theta$ is consistent, and hence, by Keisler's theorem, it has a standard model which gives the desired countable family of 1-Lipschitz functions.

\section{Final Remarks}

It is natural to ask whether there exists an uncountable (necessarily scattered) compact space $K$ such that $K^{2}$ is covered by countably many graphs of continuous functions and their inverses. Below we show that the answer is negative.

Theorem 4.1. Let $K$ be a compact Hausdorff space and let $\left\{f_{n}\right\}_{n \in \omega}$ be a family of continuous functions such that for each $n \in \omega$ the set $\operatorname{dom}\left(f_{n}\right)$ is closed in $K$ and $K \times K=\bigcup_{n \in \omega}\left(f_{n} \cup f_{n}^{-1}\right)$. Then $|K| \leqslant \aleph_{0}$.

Proof. By the Baire Category Theorem, a compact $K$ satisfying the above assertion must be scattered. Suppose the theorem is false and fix a counterexample $K$ of minimal Cantor-Bendixson rank $\lambda$. Denote by $K^{(\alpha)}$ the $\alpha$-th derivative of $K$. 
Passing to a subspace, we may further assume that $K^{(\lambda)}$ is a singleton, which we shall denote by $\infty$. Note that every closed set not containing $\infty$ is countable. Indeed, if $A \subseteq K$ is closed and $\infty \notin A$, then by compactness, $A \cap K^{(\gamma)}=\emptyset$ for some $\gamma<\lambda$. Thus the Cantor-Bendixson rank of $A$ is $\leqslant \gamma$, and therefore by the minimality of $\lambda, A$ must be countable because it satisfies the above assertion.

Let $M=\left\{f_{n}(\infty): n \in \omega\right.$ and $\left.\infty \in \operatorname{dom}\left(f_{n}\right)\right\}$ and choose $y \in K \backslash M$. Let

$$
A=K \backslash\left(M \cup\left\{f_{n}(y): n \in \omega\right\}\right) .
$$

Then $A$ is uncountable, and for each $x \in A$ there exists $k \in \omega$ such that $y=f_{k}(x)$. Find $k \in \omega$ such that the set $B=\left\{x \in A: y=f_{k}(x)\right\}$ is uncountable. Note that $\infty \in \operatorname{cl} B$, because every closed set not containing $\infty$ is countable. Thus $\infty \in \operatorname{dom}\left(f_{k}\right)$ and, by continuity, $y=f_{k}(\infty) \in M$, a contradiction.

By the result above, it is impossible to cover $\omega_{1} \times \omega_{1}$ by countably many functions that are continuous with respect to the order topology. Indeed, all these functions would be extendable onto the Cech-Stone compactification of $\omega_{1}$ which equals $\omega_{1}+1$, and therefore, adding one more function, we would obtain a countable family of continuous functions covering the square of $\omega_{1}+1$.

It is easy to see, using Sierpiński's theorem, that the one point compactification of the discrete space of cardinality $\aleph_{1}$ can be covered by countably many partial continuous functions and their inverses. Thus, Theorem 4.1 fails when we drop the assumption that $\operatorname{dom}\left(f_{n}\right)$ is closed.

\section{ACKNOWLEDGEMENTS}

The first author would like to thank Sławomir Turek for useful comments and Piotr Zakrzewski for pointing out reference [14]. Both authors would like to thank the referee for pointing out several improvements.

\section{REFERENCES}

1. U. Abraham, S. Geschke, Covering $\mathbb{R}^{n+1}$ by graphs of $n$-ary functions and long linear orderings of Turing degrees, Proc. Amer. Math. Soc. 132 (2004) 3367-3377. MR.2073314 (2005c:03090)

2. U. Abraham, M. Rubin, S. Shelah, On the consistency of some partition theorems for continuous colorings, and the structure of $\aleph_{1}$-dense real order types, Ann. Pure Appl. Logic 29 (1985) 123-206. MR801036 (87d:03132)

3. S. Geschke, A dual open coloring axiom, Ann. Pure Appl. Logic 140 (2006) 40-51. MR2224047 (2006m:03078)

4. T. JECH, Set theory. The third millennium edition, revised and expanded. Springer Monographs in Mathematics. Springer-Verlag, Berlin, 2003. MR.1940513 (2004g:03071)

5. H. J. KeISLER, Logic with quantifier "there exists uncountably many", Annals of Mathematical Logic 1 (1970) 1-93. MR0263616 (41:8217)

6. W. Kubiś, Perfect cliques and $G_{\delta}$-colorings of Polish spaces, Proc. Amer. Math. Soc. 131 (2003) 619-623. MR:1933354 (2004g:54043)

7. W. Kubiś, S. Shelah, Analytic colorings, Ann. Pure Appl. Logic 121 (2003) 145-161. MR.1982945(2004j:03055)

8. K. Kunen, Set theory. An introduction to independence proofs. Studies in Logic and the Foundations of Mathematics, 102. North-Holland Publishing Co., Amsterdam-New York, 1980. MR:597342(82f:03001)

9. K. Kunen, Forcing and differentiable functions, preprint, available at http://arxiv.org/abs/ 0912.3733v2; to appear, DOI:10.1007/s11083-011-9210-8.

10. S. Shelah, Borel sets with large squares, Fund. Math. 159 (1999) 1-50. MR.1669643 (2000i:03073) 
11. W. SierpińSki, Sur un théorème équivalent á l'hypothèse du continu, Krak. Anz. 1919, 1-3.

12. W. SierpińsKI, Sur l'hypothèse du continu $\left(2^{\aleph_{0}}=\aleph_{1}\right)$, Fund. Math. 5 (1924) 177-187.

13. W. Sierpiński, Hypothèse du continu. 2nd ed. (French), New York: Chelsea Publishing Company, 1956. MR0090558(19:829c)

14. P. Zakrzewski, On a construction of universally small sets, Real Anal. Exchange 28 (2003) 221-226. MR1973982 (2004c:03061)

Institute of Mathematics, Czech Academy of Sciences, Prague, Czech Republic and - Institute of Mathematics, Jan Kochanowski University in Kielce, Poland

E-mail address: kubis@math.cas.cz

Department of Mathematical Analysis, Charles University, Prague, Czech Republic 